\title{
Multi-target Tracking with Poisson Processes Observations
}

\author{
Sergio Hernandez ${ }^{1}$ and Paul Teal ${ }^{2}$ \\ ${ }^{1}$ Victoria University of Wellington \\ School of Mathematics, Statistics and Computer Science \\ Wellington, New Zealand \\ sergio.hernandez@mcs . vuw .ac.nz \\ ${ }^{2}$ Victoria University of Wellington \\ School of Chemical and Physical Sciences \\ Wellington, New Zealand \\ paul.teal@vuw.ac.nz
}

\begin{abstract}
This paper considers the problem of Bayesian inference in dynamical models with time-varying dimension. These models have been studied in the context of multiple target tracking problems and for estimating the number of components in mixture models. Traditional solutions for the single target tracking problem becomes infeasible when the number of targets grows. Furthermore, when the number of targets is unknown and the number of observations is influenced by misdetections and clutter, then the problem is complex.

In this paper, we consider a marked Poisson process for modeling the time-varying dimension problem. Another solution which has been proposed for this problem is the Probability Hypothesis Density (PHD) filter, which uses a random set formalism for representing the time-varying nature of the state and observation vectors. An important feature of the PHD and the proposed method is the ability to perform sensor data fusion by integrating the information from the multiple observations without an explicit data association step. However, the method proposed here differs from the PHD filter in that uses a Poisson point process formalism with discretized spatial intensity.

The method can be implemented with techniques similar to the standard particle filter, but without the need for specifying birth and death probabilities for each target in the update and filtering equations. We show an example based on ultrasound acoustics, where the method is able to represent the physical characteristics of the problem domain.
\end{abstract}

Keywords: Bayesian inference, marked Poisson process, multi-target tracking, sequential Monte Carlo methods, particle filters.

\section{Introduction}

Tracking multiple objects with multiple sensors is a problem where the dimensionality of the posterior distribution is evolving in time. Such problems are called

D. Mery and L. Rueda (Eds.): PSIVT 2007, LNCS 4872, pp. 474-483 2007.

(C) Springer-Verlag Berlin Heidelberg 2007 
"transdimensional" [1]. Furthermore, the procedure of making inference about the current state of the system involves the estimation of non-linear and nonGaussian functions. State space models provides a sound framework for building a probabilistic model from a sequence of observations contaminated with noise. However, not all the solutions for fixed dimensionality are directly applicable to variable dimension models. Transdimensional problems are common in inverse problems, signal processing and statistical model selection. In this paper, a method for handling such problems is provided, and multiple target tracking is to be considered as an example of such a problem. The method is then applied to parameter estimation for acoustic sources.

Sequential Monte Carlo (SMC) methods have been proposed [2] as a framework for dealing with non-linear and non-Gaussian target tracking problems. These methods use the Bayesian approach for simulating several hypotheses taken from an easy-to-sample proposal distribution and weight them using their likelihood with the observed data. The most standard implementation of a SMC method, called the particle filter has demonstrated good performance in several domains as well as having theoretical convergence properties [4].

In the multiple target tracking problem, the number of targets may be timevarying and unknown. The number of measurements or observations may also be time varying. The traditional approaches for performing measurement-target data association include multiple hypothesis tracking (MHT) and joint probabilistic data association (JPDA), which separates the problem into different sub levels. These approaches use thresholding heuristics to associate each measurement to an existing target, create a new target, merge measurements or mark them as false alarms [5].

Particle filtering has been successfully used for multiple target tracking [6], where the data association problem is formulated in a non-explicit way using a joint multiple-target probability density which comprises the estimation of the state of several targets, the number of the targets and the likelihood of the observations with each of them. More recently, the Probability Hypothesis Density (PHD) filter has been proposed to deal with the problem of the unknown number of targets, using random sets theory [7. The PHD filter propagates the first moment of the multi-target posterior distribution, which (for known dimensionality) results in an analytically tractable Bayesian solution for the multi-target posterior. If the multi-target likelihood can be approximated as a Poisson process, then the integral of the PHD in any region of the space gives the expected number of targets on that region 8 .

In this paper, we work further on the spatial Poisson process model presented in 910] for tracking extended targets. The model is able to perform seamlessly in the multi-target tracking problem as well as solving the data association problem. We propose a SMC approximation to the conditional spatial intensity of the point process. The approximation uses a discretization of the spatial intensity and a modification of the standard particle filter, to take in account spontaneous targets birth. The model is tested with unthresholded physical measurements, where the number of measurements is dependent on the unknown number of 
targets. An example in the bearings-only tracking problem is provided, as well as an example in range estimation from ultrasound acoustics. The example shows that the model is well suited to characterize physical features from acoustic measurements in uncertain environments.

The paper is structured as follows. In Section 2 we outline the PHD filter. In Section 3 the spatial Poisson process model is presented, and then in Section 4 we present a new sequential Monte Carlo method for an unknown number of targets. Section 5 provides examples that show the feasibility of the model in two different problems. Section 6 present the conclusions.

\section{PHD Filter}

First-order moment of a multi-target posterior distribution: The PHD filter represents the first moment of the intensity function $\lambda$ of the multi-target posterior distribution. For any measurable subset $S$ of the space of possible random sets, the regions of high intensity indicate the actual locations of the targets, and the total mass of the same function can provide an estimate of the number of $\operatorname{targets} N$ :

$$
E[N(S)]=\int_{S} \lambda(x) d x
$$

The PHD filter operates using the following procedure:

1. Filtering:

$$
\lambda_{t}\left(x_{n, t}\right)=\int_{S} f\left(x_{n, t} \mid x_{n, t-1}\right) P_{s}\left(x_{n, t-1}\right) \hat{\lambda}_{t-1}\left(x_{n, t-1}\right) d x_{n, t-1}+\gamma\left(x_{n, t}\right)
$$

where $f\left(x_{n, t} \mid x_{n, t-1}\right)$ represents the probability density of motion of the $n$th single target from state $x_{n, t-1}$ to state $x_{n, t}$ at time $t, P_{s}\left(x_{n, t}\right)$ is the probability of survival for the $n$th target at time $t$, and $\gamma()$ is the birth probability.

2. Update:

$$
\hat{\lambda}_{t}\left(x_{n, t}\right)=\left[1-P_{d}\left(x_{n, t}\right)+\sum_{m=1}^{M_{t}} \frac{\psi_{m, t}\left(x_{n, t}\right)}{<\psi_{m, t}, \lambda_{t}>+\kappa\left(z_{m, t}\right)}\right] \lambda_{t}\left(x_{n, t}\right)
$$

where

$$
\begin{gathered}
\psi_{m, t}(x)=P_{d}(x) L\left(z_{m, t} \mid x\right) \\
<\psi_{m, t}, \lambda>\triangleq \int_{S} \psi_{m, t}(x) \lambda(x) d x
\end{gathered}
$$

and where $P_{d}\left(x_{n, t}\right)$ is the probability of detection, $\kappa()$ is the clutter rate, $L\left(z_{m, t} \mid x\right)$ is the likelihood of the $m$ th observation and $M_{t}$ is the total number of observations at time $t$. 
SMC implementation of PHD: The particle filter implementation for the PHD recursion was proposed by 1112 as a method for simulating the conditional expectation of the unknown number of targets given the current observations. The SMC implementation of the PHD approximates the intensity function $\lambda(x)$ with a set of particles. Further results on the convergence properties for the particle implementation were given in [13] and an extension to Gaussian mixture models for solving the integral for the PHD recursion [14].

\section{$3 \quad$ State Space Model with Poisson Process Observations}

A marked point process is a random collection of events that happens in time and some other dimensions (in this case space) [15. The number of observations $M_{t}$ received in the interval $\Delta_{t}=[t-1, t)$ can be written as a marked point process with intensity $\lambda(A, t)$ dependent on time and the spatial parameter $A$.

$$
p\left(M_{t} \mid \lambda(A, t)\right)=\lambda(A, t)^{M_{t}} \frac{\exp [-\lambda(A, t)]}{M_{t} !}
$$

The mean rate of observations $\lambda(A, t)$ can be decomposed into a rate for clutter components with homogeneous (i.e., constant) spatial intensity function $\lambda_{c}$, and an intensity component for each of the $N_{t}$ targets:

$$
\lambda(A, t)=\left(\lambda_{c}+\sum_{n=1}^{N_{t}} \lambda_{n}(A)\right) \Delta_{t}
$$

The spatial intensity $\lambda(A)$ can also be written in terms of the spatial posterior distributions of the targets $X_{t}=x_{1}, . ., x_{N_{t}}$ and the observations $Z_{t}=z_{1}, . ., z_{M_{t}}$.

$$
\lambda_{n}(A)=\int_{A} \lambda\left(z \mid x_{n}\right) d z
$$

So the conditional likelihood of the observations on the targets states can be written as:

$$
\begin{aligned}
p\left(Z_{t} \mid X_{t}\right) & =\frac{\exp (-\lambda(A, t))}{M_{t} !} \prod_{m=1}^{M_{t}} \lambda\left(z_{m} \mid X\right) \\
& =\frac{\exp (-\lambda(A, t))}{M_{t} !} \prod_{m=1}^{M_{t}}\left[\lambda_{c}+\sum_{n=1}^{N_{t}} \lambda_{n}\left(z_{m} \mid x_{n}\right)\right]
\end{aligned}
$$

\section{SMC Approximation for the Spatial Poisson Process Model}

We decompose $A$ using some suitable spatial discretization into $K$ disjoint unions (here called bins) so as to transform the problem into counting in each bin. The 
intensity function $\lambda_{n}\left(z_{k} \mid x_{n}\right)$ defines the rate of observations of a particular target $x_{n}$ in a measurement bin $k$. Given that the overall intensity is a superposition of a discrete number of Poisson processes, it is difficult to calculate the intensity for a single target. A possible solution is to approximate the intensity with a quantity proportional to the likelihood of the $n$th target and inversely proportional to the number of observations in that bin.

$$
\int_{A} \lambda\left(z \mid x_{n}\right) d z=\sum_{k=1}^{K} p\left(z_{k} \mid x_{n}\right) / m_{k}
$$

A particle filter model is then used for propagating the conditional spatial intensity of each target, which can be used by the multi-target likelihood (9) for calculating the posterior distribution of the observations. The conditional spatial intensity can be approximated with the update and filtering equations of the standard SMC methods, but in order to take into account targets appearing and dissapearing we use resampling without replacement. In this setup, only $r$ particles survive and the remaining samples gives birth to new samples with probability $b(x)$.

\section{$5 \quad$ Examples}

In this section, two examples are provided for the spatial Poisson process model.

Multi-target bearings-only tracking: The bearings-only tracking problem is a non-linear Gaussian problem where a passive observer is taking measurements of the angle for which a target is moving in two dimensions. If the number of targets is dynamically changing, then the multi-target Gaussian likelihood becomes intractable and the optimal filter needs to resort in additional techniques for estimating the system state. Figure 1 shows the target trajectories for this example. Each target is represented by its 2-D position and velocity $\boldsymbol{x}_{n, t}=\left(x_{n, t}, y_{n, t}, d x_{n, t}, d y_{n, t}\right)$. The target dynamic model can be written as $\boldsymbol{x}_{n, t}=G \boldsymbol{x}_{n, t-1}+\eta_{t}$. The state equation innovation is a bivariate zero-mean Gaussian process $\eta \sim \mathcal{N}\left(0, \sigma^{2} I\right)$. The observation model is the non-linear function of the position $z_{n, t}=\tan ^{-1}\left(x_{n, t} / y_{n, t}\right)+\alpha_{t}$.

A finite hidden Markov model can be used for the observed number of measurements given an unknown number of targets. This model can represent the probability of a change in dimension given the history of the number of targets and observations The example shows a model jump from 5 targets to 6 targets, with constant clutter probability $\lambda_{c}=1$ and probability of detection $P_{d}=1$ for each state. Figure 2 shows the time-varying dimensionality of the state and observations.

Figure 3 shows the superimposed observations received at each time step. The number of observations received is a point process with inhomogeneous spatial intensity, and there is not enough information for an explicit measurement-totrack data association. 


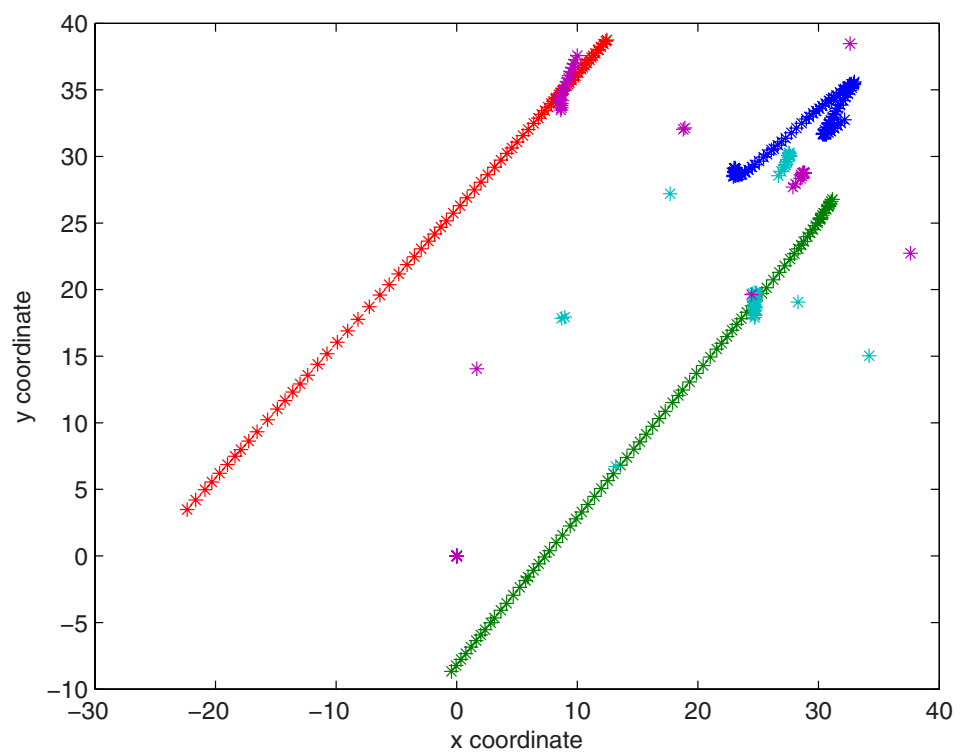

Fig. 1. Multi-target Tracking Model

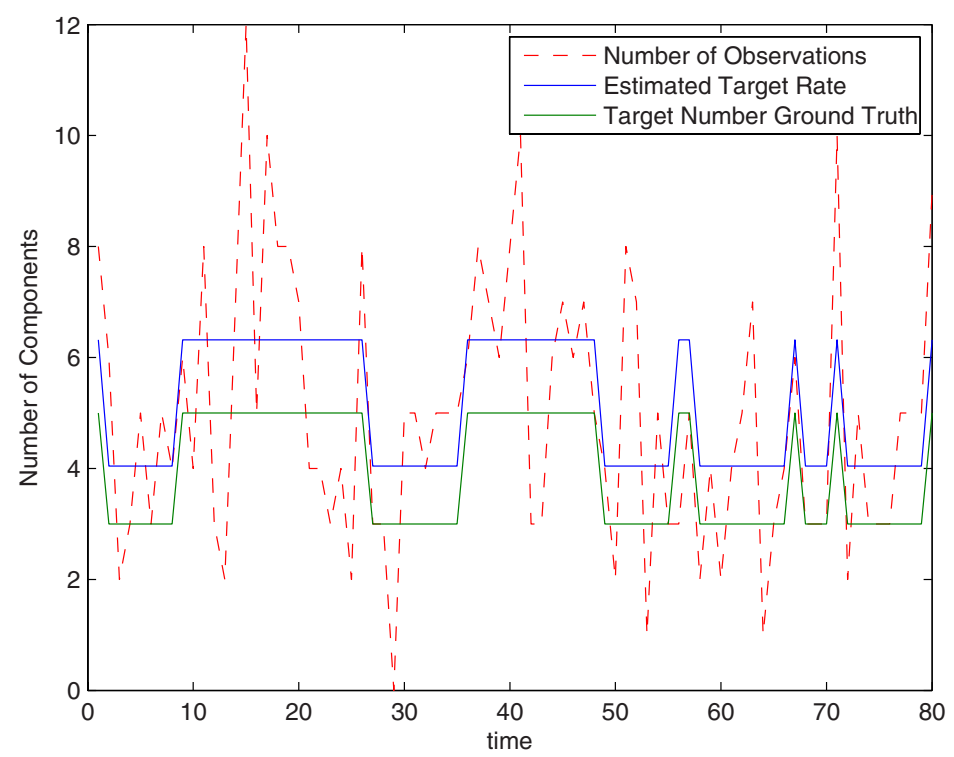

Fig. 2. Poisson Hidden Markov Model

The particle filter model uses 100 particles to represent the intensity function $\lambda\left(z \mid x_{n}\right)$. The particles are resampled without replacement. Thus the low weighted particles are killed but not replaced with copies of the best weighted particles. 


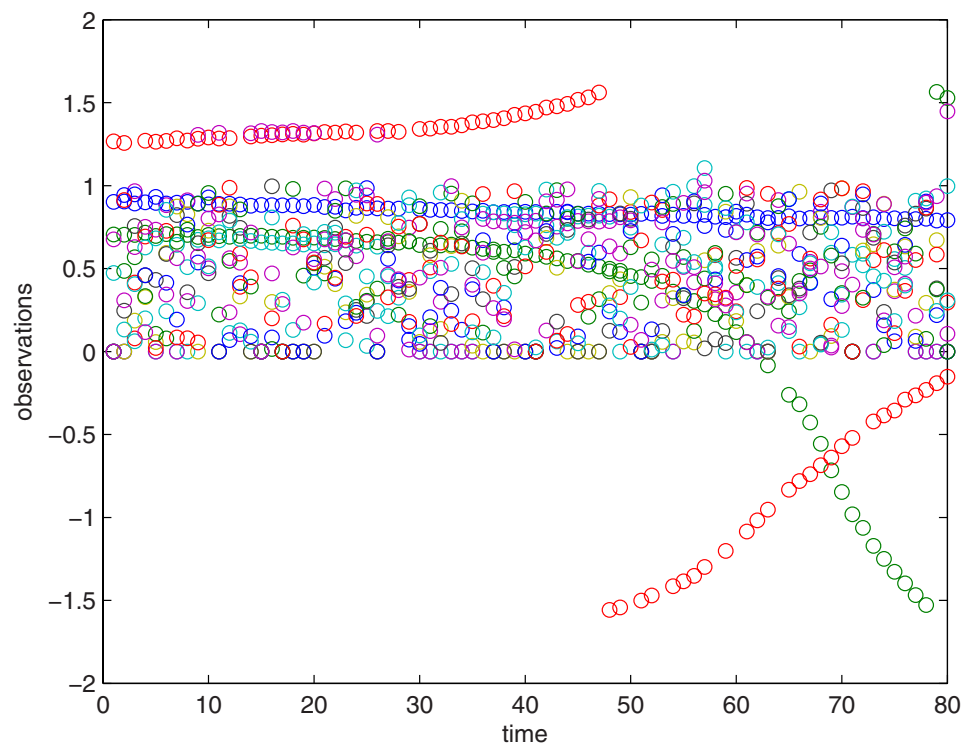

Fig. 3. Spatial Poisson Process

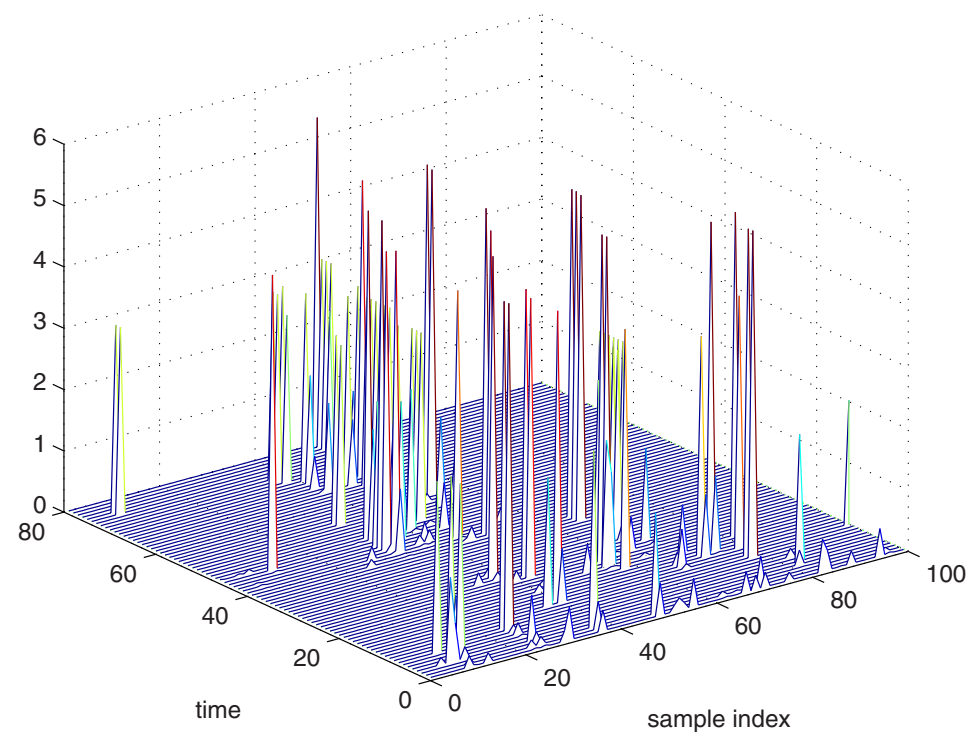

Fig. 4. Conditional Intensity Monte Carlo Estimate

They are used to give birth to new samples. The particles are used to represent a weighted conditional intensity. Figure 4 shows the evolution of the particle system. 

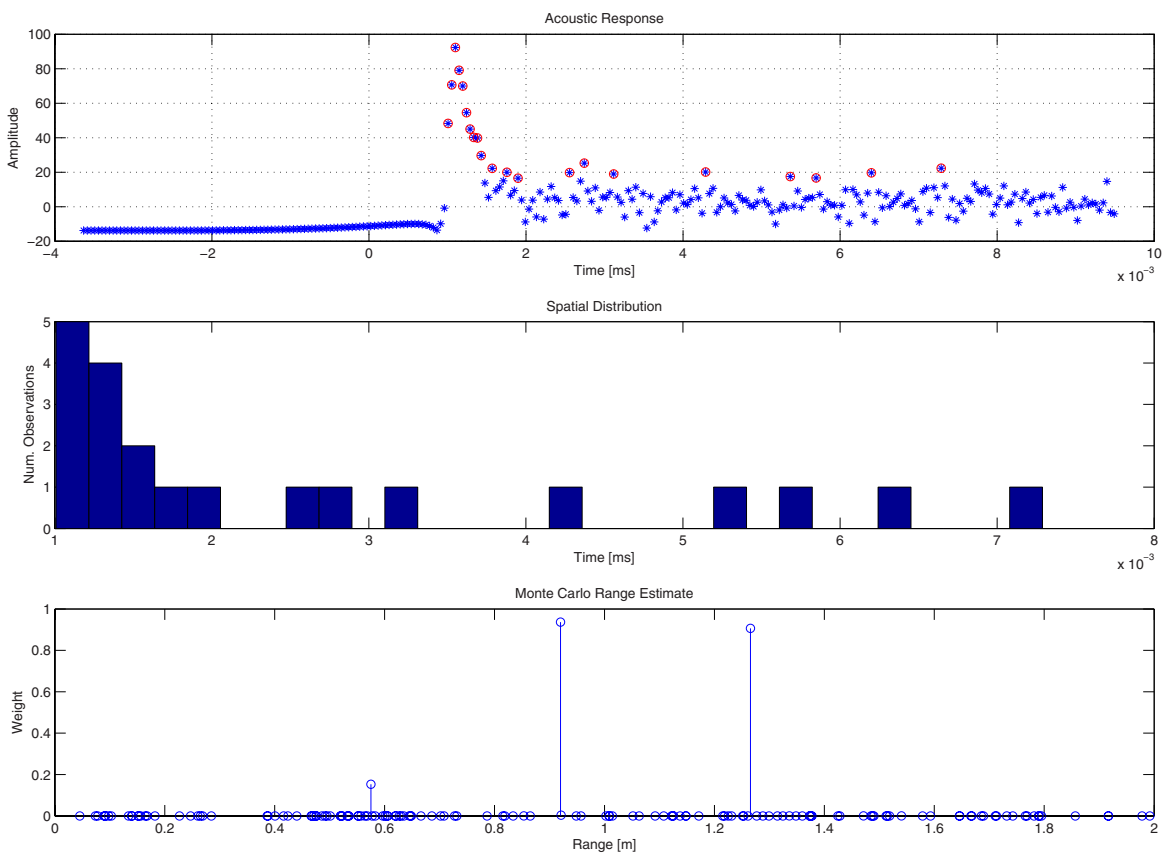

Fig. 5. Single Channel Acoustic Data (Channel 1)
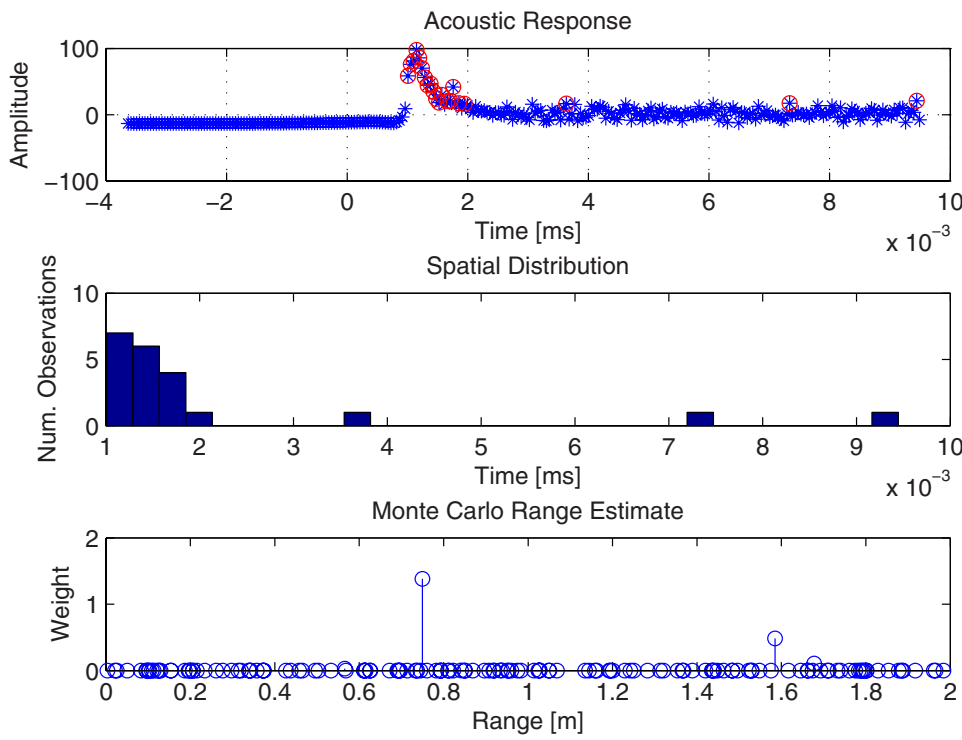

Fig. 6. Single Channel Acoustic Data (Channel 10) 
Range estimation from acoustic measurements: The location of targets using acoustic measurements in reverberant environments is difficult to characterize because of the multi-path propagation. Estimating range from time-delay measurements from multiple reflecting objects using an array of sensors can be a challenging problem when the model dimension is unknown. Time-delay measurements are a superposition of the first reflection of a target, the multi-path reflections, and the background noise.

In this example, ultrasound measurements are received by an array of sensors. A known signal is propagated in the air, and the received acoustic energy is represented by the complex response of the sensors. The observations here correspond to any samples above a threshold such as the variance of the complex signal. The range of the target is represented as a function of the time-delay of arrival of the wavefront for the first reflection of each target.

A Monte Carlo importance sampling method is used for estimating the range of the target for each measured time delay. The range is calculated as $r=c t_{d}+w$, where $c$ is the velocity of sound propagation in air, $w \sim \mathcal{N}\left(0,10^{-6}\right)$ and $t_{d}$ is the measured time delay.

Successful target detection is shown in Figure 6.

\section{Conclusion}

This paper has presented a SMC approximation to the spatial Poisson process model for multi-target tracking problems. The model uses a Poisson point process in a multidimensional space, therefore it can be thought as being part of Mahler's Finite Set Statistics framework. The formulation presented does not make use of an explicit birth and death strategy, but uses a modified sequential Monte Carlo method that performs resampling without replacement. In that way, new particles are born in the place of the resampled particles, allowing a non-explicit birth and death formulation.

The case of multiple target tracking is illustrated as a motivating example. Although the method proposed has no apparent improvement on the PHD recursion for the tracking problem, the example in acoustic parameter estimation has shown the feasibility of the model for representing the measurement superposition due to reverberation. This is an important feature that makes the model practical to use with unthresholded physical measurements. On the other hand, it shares the problems of the PHD filter of being highly dependent on the SNR ratio and being difficult to interpret. Further work will be done on calculating the target state estimate and comparing the results with the ground truth data. For the acoustic problem presented, new results will be extended to multi-channel observation data.

\section{Acknowledgement}

The authors would like acknowledge Industrial Research Limited for providing the facilities for capturing the data used in this paper. 


\section{References}

1. Sisson, S.A.: Transdimensional Markov chains: A decade of progress and future perspectives. Journal of the American Statistical Association 100(471), 1077-1089 (2005)

2. Gordon, N.J., Salmond, D.J., Smith, A.F.M.: Novel approach to nonlinear/nonGaussian Bayesian state estimation. IEEE Proceedings 2(140), 107-113 (1993)

3. Liu, J.S., Chen, R.: Sequential Monte Carlo methods for dynamic systems. Journal of the American Statistical Association 443(93), 1032-1044 (1998)

4. Doucet, A., de Freitas, N., Gordon, N.: Sequential Monte Carlo methods in practice. Springer, New York (2001)

5. Blackman, S., Popoli, R.: Design and Analysis of Modern Tracking Systems. Artech House, Norwood (1999)

6. Hue, C., Le Cadre, J.P., Perez, P.: Sequential Monte Carlo methods for multiple target tracking and data fusion. IEEE Trans. on Signal Processing 2(50), 309-325 (2002)

7. Mahler, R.P.S.: Multitarget bayes filtering via first-order multitarget moments. IEEE Transactions on Aerospace and Electronic Systems 39(4), 1152-1178 (2003)

8. Mahler, R.P.S.: Statistical Multisource-Multitarget Information Fusion. Artech House, Norwood (2007)

9. Godsill, S., Li, J., Ng, W.: Multiple and extended object tracking with Poisson spatial processes and variable rate filters. In: IEEE International Workshop on Computational Advances in Multi-Sensor Adaptive Processing, IEEE Computer Society Press, Los Alamitos (2005)

10. Gilholm, K., Godsill, S., Maskell, S., Salmond, D.: Poisson models for extended target and group tracking. In: Signal and Data Processing of Small Targets 2005, SPIE (2005)

11. Vo, B.N., Singh, S., Doucet, A.: Sequential Monte Carlo methods for multitarget filtering with random finite sets. IEEE Transactions on Aerospace and Electronic Systems 41(4), 1224-1245 (2005)

12. Sidenbladh, H.: Multi-target particle filtering for the probability hypothesis density. In: Proceedings of the Sixth International Conference of Information Fusion (2003)

13. Clark, D.E., Bell, J.: Convergence results for the particle PHD filter. IEEE Transactions on Signal Processing 54(7), 2652-2661 (2006)

14. Vo, B., Ma, W.: The Gaussian mixture probability hypothesis density filter. IEEE Transactions on Signal Processing 54(11), 4091-4104 (2006)

15. Daley, D., Vere-Jones, D.: An Introduction to the Theory of Point Processes. Elementary Theory and Methods, vol. I. Springer, New York (2003) 